\title{
Goslings of gay geese
}

\section{Jared M. Diamond}

Homosexual female pairs of wild birds - already reported for four gull, one tern and one goose species - have aroused much interest ${ }^{1-7}$. Does the phenomenon reflect a hormonal or behavioural idiosyncracy of the homosexual females that distinguishes them from heterosexual females, one that produces no evolutionary benefit (no offspring)? Or is it instead an adaptation compatible with reproduction and favoured by natural selection under conditions of male scarcity? A recent study by Quinn $e t$ al. ${ }^{1}$ demonstrates the feasibility of the latter interpretation.

Recognition of the phenomenon stemmed from the observation that up to 14 per cent of western gull clutches on Santa Barbara Island, California, contained twice as many eggs as do normal clutches ${ }^{2}$. Although this observation might in principle have arisen from other causes (such as polygynous females sharing a nest, brood parasitism or egg dumping), the prevalent cause turned out to be pairs consisting of two females, both of whom laid eggs. Like male/female pairs of gulls, paired females often make lasting bonds and nest with each other in successive years in the same colony, even at the same nest site $^{2.3}$. Homosexual female pairs of western gulls practise the same courtship and territorial behaviour as do heterosexual pairs, except that courtship feeding is rare and only three homosexual pairs

100 years ago From Nature 40, 280; 18 July 1889.

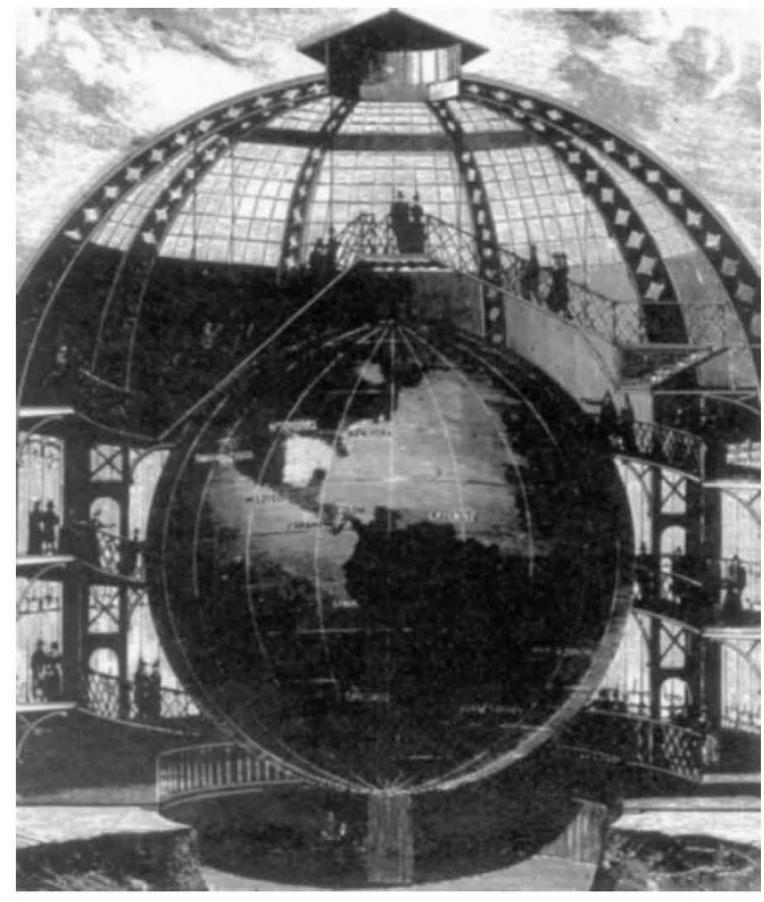

One of the great attractions of the Paris Exhibition is a great terrestrial globe, one millionth of the actual size of the earth. exhibited attempted copulation ${ }^{2}$

Homosexual pairing is often associated with a shortage of males in the nesting colony ${ }^{4,5}$. The ratio of males to females collected since 1950 among adult western and herring gulls is only $0.42-0.43$, for example, because of selective post-fledging mortality of males ${ }^{5}$. It is interesting that above-average sized clutches have been

\section{IMAGE UNAVAILABLE FOR COPYRIGHT REASONS}

Natural herstory - the lesser snow goose. (Courtesy of Dr Fred Cooke.)

regularly observed in these two species only since 1950 ; before 1950 , the sex ratio was closer to 1.0. Experimental removal of many males from California and ringbilled gull colonies resulted in a threefold increase in frequency of 'supernormal' clutches compared with control colonies ${ }^{6}$.

For homosexual pairing in gulls to be explicable by natural selection at the individual level, the female must somehow become fertilized and succeed in rearing offspring. Western gull female pairs provided experimentally with fertile eggs from other nests do incubate, feed and fledge young with virtually normal success rates ${ }^{2}$. Among the eggs found naturally in supernormal clutches, many are infertile but some are fertile. How do paired females become fertilized?

Quinn et al. ${ }^{1}$ now report results of a restriction-fragment-length polymorphism analysis to determine parentage of goslings hatched by a female pair of lesser snow geese on Hudson Bay, Canada. When discovered, the nest contained eight eggs, twice the normal clutch size for the species. Seven of the eggs hatched. Both females defended the goslings, and removal of one female led the other to give loud 'mate calls' typical of male/female pairs. The genetic analysis indicates that goslings 1,2 and 3 stemmed from one of the females fertilized by one male; goslings 4,6 and 7 stemmed from the other female fertilized by a different male; and gosling 5 could have stemmed from either female but had to have been sired by yet a third male. Thus, both females had become fertilized independently and were not the co-widows of one deceased or absent polygynous male.

This is the first observation of female pairing in lesser snow geese, a species for which the sex ratio is not known to be skewed. Although the advantage of female pairing is thus uncertain for the geese, its adaptive value now becomes clear for the gull colonies having a shortage of males. One gull alone is incapable of rearing young because other gulls destroy the clutch when the single parent leaves to feed. Normal nests of male/ female pairs are always attended by one parent. In a nominally monogamous colony a male deficit means that some females cannot obtain male mates. But any female can still become fertilized (at least in gulls) by some nominally monogamous male, as mated males seek to copulate with females other than their mate. Once fertilized, two females can rear the young almost as well as one female plus one male.

These observations raise the perennial question of why males exist at all at a sex ratio near 1.0. After a male gull has contributed semen, he appears to play almost no role that a female cannot play equally well. It is true that female gulls do not provide each other with the courtship feeding that male gulls provide their mates, with the result that eggs of homosexual female pairs are smaller and may have poorer posthatching survival than do eggs of heterosexually paired females ${ }^{2}$. However, if long-term reproductive success per egg is at least 50 per cent of normal, homosexual pairs would still have a higher reproductive output per individual than do heterosexual pairs.

It is also true that, in species whose males are much bigger than females (unlike male gulls), males are useful for protecting the young. Yet other males themselves are one of the main threats in the first place. Further study of homosexually paired female birds may help clarify what, if anything, males are good for - in an evolutionary sense, of course.

Jared M. Diamond is a professor of Physiology at the University of California Medical School, Los Angeles, California 90024-1751, USA.

1. Quinn, T.W. et al. Auk 106, 177-184 (1989).

2. Hunt, G.L. \& Hunt, M.W. Science 196, 1466-1467 (1977).

3. Kovacs, K.M. \& Ryder, J.P. Auk 98, 625-627 (1981)

4. Hunt, G.L. et al. Auk 97, 473-479 (1980).

5. Conover, M.R. \& Hunt, G.L. Wilson Bull. 96, 619-625 (1984)

6. Conover, M.R. \& Hunt, G.L. Condor 86, 472-476 (1984)

7. Conover, M.R. Condor 86, 467-471 (1984). 